\title{
A Study of Cloud Distributions using Reflected Radiance Measurements from the ATS Satellites ${ }^{1}$
}

\author{
Alfred J. Stamm and Thomas H. Vonder Haar \\ Space Science \& Engineering Center, University of Wisconsin, Madison \\ (Manuscript received 12 November 1969, in revised form 27 January 1970)
}

\begin{abstract}
Reflected radiance measurements from the multicolor spin-scan cloud camera on Applications Technology Satellite III are used to determine the percentages of selected areas of the earth that are cloud-free. The areas chosen are meteorologically active and represent common cloud patterns. Use of several data unit sizes shows how the observed percent clear area decreases with decreasing spatial resolution of a simulated sensor. Methods of determining a cloud-no cloud threshold are discussed. The change of cloud cover over a period of a few hours is examined. It is found that clouds smaller than the instantaneous field of view are of ten not recognized as clouds and therefore tend to affect the interpretation of spacecraft camera measurements. The results of this investigation are used to suggest the optimum spatial resolution for radiometrically sounding the atmosphere from a geosynchronous satellite using an instrument described in the report.
\end{abstract}

\section{Introduction}

There is increasing interest today in detecting, from satellites, radiation that is reflected or emitted from the earth. In order to see the earth, a satellite must look between the clouds, but there is very little information as to the size and frequency of the cloudless areas.

One need for this information is to specify the optimum field of view of a radiometer designed to measure radiances from which the vertical temperature profile can be calculated (COSPAR, 1969). In order to acquire a temperature profile down to the ground, cloudless areas are preferred, but the most interesting weather occurs where there are clouds. Since a number of readings are needed for "good statistics," especially from a geosynchronous orbit, the problem of how close one can sound to baroclinic meteorological regions translates to the problem of what percentage of the area near various cloud patterns is cloudless. Also needed is information on the size of the cloudless areas, or rather what instrument spatial resolution is needed to see between the clouds. This is necessary because a trade-off must be made between the spatial resolution and the sensitivity of the instrument. More will be said about sounding in a later section.

Scientists in the Earth Resources Technology Satellite (ERTS) program are interested in looking at certain areas of the earth with various sun angles and at various times of the year. Salomonson (1969) gives an extensive list of references on cloud statistics. Before a viewing program can be outlined, information is needed on the clouds covering these areas at the times of interest, especially areas that are cloudy most of the time. Such

\footnotetext{
${ }^{1}$ Research sponsored by the Laboratory for Atmospheric and Biological Sciences, NASA, under Contract NAS 5-11542.
}

questions as: "Can the information be obtained by looking between the clouds, or are cloudless days needed?" and "What is the likelihood of a cloud cover at various times of the day and year?" will need to be answered.

A third possible need for cloud statistics data could be in determining the types of clouds by correlating the cloud radiance and variance in radiance with the amount of cloudless area. This approach may be called a quantitative nephanalysis. The present study is an initial attempt to derive information on cloud coverage specifically needed for the vertical temperature sounding problem. Portions of the work presented apply equally well to other applications of radiance measurements from a geosynchronous platform.

\section{Description of data}

Among the best cloud cover data sources available today are the Applications Technology Satellite (ATS) spin-scan cloud camera pictures from ATS-I and ATS-III. In these experiments the "camera" consists of a photomultiplier behind the optics of a small telescope. The pictures are made as the instantaneous field of view (IFOV) of the camera telescope $(0.1 \mathrm{mrad})$ scans across the earth, while the satellite in geosynchronous obrit is spinning on an axis parallel to the earth's axis (Thomsen et al., 1969). After each scan, while the camera is pointed away from the earth, the camera is stepped down by an amount slightly greater than the angular field of view so that a new scan is made parallel to and just south of the previous scan. On ATS-III, 2400 scans north to south comprise a complete picture of the earth. At the nominal 100-rpm spin rate this means a picture can be made in slightly less than one- 


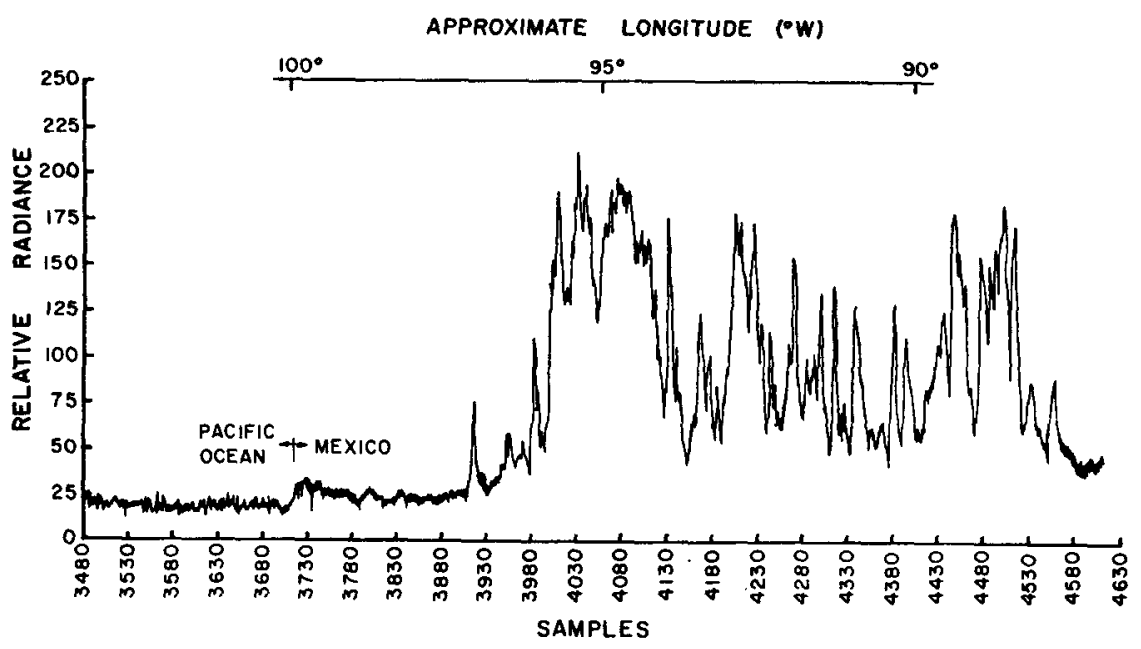

FIG. 1. Radiance level plot for part of a single scan line for 20 January 1968, 1911:31 GMT, green channel, latitude about $17 \mathrm{~N}$.

half hour. The analog camera output of the $20^{\circ}$ segment which comprises a scan across the earth is digitized into 8192 samples of 2.35 samples per IFOV. Each sample is made up of 8 bits giving a dynamic range of 256 radiance levels. Vonder Haar (1969) discusses the relationship between the digitized counts (relative radiance) and the reflected solar radiance measured by the photomultiplier.

Fig. 1 shows a radiance level plot for part of a single scan line. ATS-III pictures have three colors-blue, green and red-but only the green channel (0.48-0.58 $\mu \mathrm{m})$ is used in the work reported in this article. This figure shows very well both the high dynamic range and high spatial resolution of the ATS data.

Since there are more than two samples per field of view, each sample does not necessarily represent new information (i.e., the scan samples overlap). For this reason and also in order to work with a more square basic unit size, the values of three adjacent samples are averaged. Using the scan step size rather than the field of view, the size of the basic unit is roughly $4.6 \mathrm{~km}$ north to south at nadir and slightly less east to west. These basic units are then combined so that 1, 4, 9, 16,25 or 36 go into a square data unit which is the area corresponding to the simulated spatial resolution or instantaneous field of view of a future instrument. A grid or field is the square area over which the computations are made and is 60 basic units across for the former and 100 for the latter. This corresponds to 276 and $460 \mathrm{~km}$ at nadir, respectively. The former size was chosen because it is divisible by $1,2,3,4,5$ and 6 , and the latter size was used for parallel work being done on the same ATS picture (Hanson, 1969). Note that these areas are $48 \%$ and $132 \%$, respectively, of the area of a basic Global Atmospheric Research Program (GARP) grid mesh (GARP, 1969). A matrix is that combination of grids or fields covering the particular cloud pattern of interest.

\section{Methods of simulating the IFOV}

In terms of the ATS data, the problem is stated as follows: What percentage of the different size data units within a given grid or field are cloud-free? Two methods have been used to treat this problem.

The first method, called Method A, approximates an instrument which uses its entire IFOV to compare with a cloud-no cloud threshold (Th). This threshold, which is given in terms of a radiance level, will be discussed further in the next section. Method A uses the square data units and takes the average radiance of all the samples within the data unit to compare with the threshold. That is, each data unit is clear that satisfies the equation

$$
\mathrm{Th}-\sum_{i=1}^{n} S_{i} / n>0
$$

where $S_{i}$ is the radiance value of the $i$ th sample within the data unit, and $n=3,12,27,48,75$ or 108 depending on which data unit size is used. The observed percent clear area is then the percentage of the data units within a grid or field that satisfy the above equation (i.e., are entirely clear). This percentage will change with spatial resolution simulated by changing the data unit size. It should be noted here that the percent clear area observed at a given resolution is not the actual percent clear area (i.e., infinite resolution).

Fig. 2 shows, when using Method A, that the observed percent clear area does not decrease significantly with deteriorating spatial resolution (i.e., increasing data unit size); in some cases it even increases. This is misleading because, as the IFOV increases, small clouds are more likely to be found within the IFOV. In other words, viewing between some of the clouds may no longer be possible, and the observed percent clear area should decrease. 


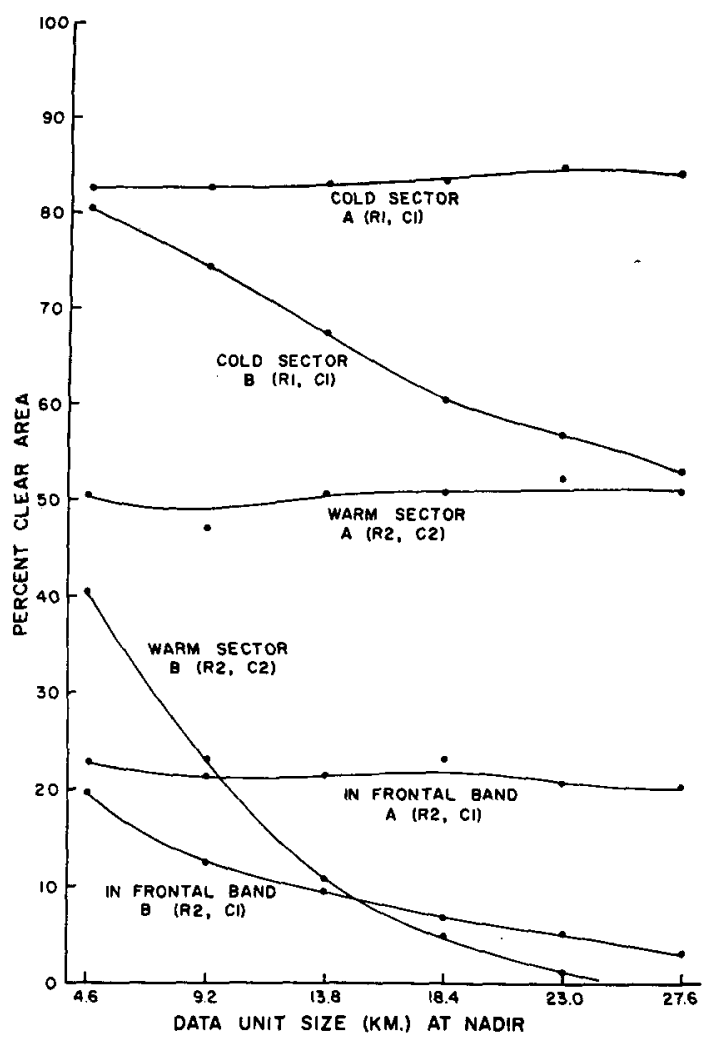

FIG. 2. Percent clear area for various data unit sizes of an extratropical front over northwestern United States for 23 April 1968, 1714:10 GMT, matrix 3. A and B refer to the method (see text) and row and column locate grid within matrix.

The reason for this difficulty is as follows. When averaging over a large data unit, a small cloud may be obscured since a small, fairly dark cloud can average with dark land around the cloud to equal a radiance level comparable to bright land. (In ATS pictures, land and ocean are generally darker than clouds.) As a result, clouds smaller than the IFOV may not be detected.

This difficulty is significant for the following reason. Based on experience with the relatively low spatial resolution temperature sounders on Nimbus-III, Smith $^{2}$ estimates that $10 \%$ cloudiness within the IFOV is enough to make a temperature profile below the cloud level inaccurate. Smith (1968) also shows that the effect of such partial cloudiness can be overcome only with the aid of special cloud statistics or additional information about the atmospheric temperature profile.

To alleviate the difficulty of averaging over large data units (Method A), a second method, B, was designed. It labels data units as cloudy even if only one sample is over the threshold. Method B, therefore, simply changes every radiance level over the threshold to one million and then proceeds as in Method A. The problem of calling small dark clouds "clear" is thus almost eliminated because the averaging is done over a much smaller

${ }^{2}$ William L. Smith, NESC, ESSA, private communication. area, i.e., an ATS sample. This method approximates an instrument that uses another channel with an IFOV smaller than the sounding channels (e.g., a cloud mapping channel used to determine cloud velocities) to determine whether or not clouds are actually present. In other words, a data unit is now observed as clear if it satisfies the equation

$$
\mathrm{Th}-\sum_{i=1}^{n} r_{i} / n>0
$$

where

$$
\left.\begin{array}{l}
r_{i}=S_{i}, \text { if } \mathrm{Th} \geq S_{i} \\
r_{i}=1,000,000, \text { if } \mathrm{Th}<S_{i}
\end{array}\right\},
$$

remembering that $S_{i}$ ranges from 0 to 255 .

In comparing Method A with Method B (Fig. 2), note that not only is the decrease in percentage clear area with resolution greater for Method B than for Method $A$, but also that the percentage clear area at $4.6-\mathrm{km}$ resolution is less for Method B. The latter occurs because Method A averages over three samples while Method B compares the value of each sample with the threshold. This gives some insight as to how an actual instrument (including, of course, the ATS sensor ${ }^{3}$ ) may erroneously flag an area as cloud-free when clouds smaller than the IFOV are present. Since most instruments approximate Method A, i.e., they average the radiances within their field of view, an instrument to be used for radiometrically sounding the atmosphere should be designed so that the cloud recognition is made at a higher resolution than the sounding.

Fig. 3 shows a map of the clear areas at three spatial resolutions using Method $B$. It depicts a case of cumulus and stratus clouds in the subtropics. If this region had been treated with Method A, not all of the $9.2-\mathrm{km}$ resolution cloudy area would have been within the $18.4-\mathrm{km}$ resolution cloudy area nor the $4.6-\mathrm{km}$ resolution area within the $9.2-\mathrm{km}$ resolution area. The remainder of this study uses Method B.

\section{Threshold determination}

As mentioned before, each element has 256 possible radiance levels. Clouds are almost always brighter than either land or water but the question is: "How much brighter?" Cirrus and other type clouds may be quite dark (poor reflectors). This makes it difficult to determine a cloud-no cloud threshold.

A combination of two methods is used in this paper to select thresholds. First a plot of relative radiance vs sample number is made for a given line. By locating this line on the corresponding picture, the samples which represent cloudless land and ocean values can be determined. The upper values of clear land and ocean are then extracted from the line plot. Fig. 1 shows a typical

\footnotetext{
${ }^{3}$ W. Shenk, GSFC, NASA, is currently comparing the ATS radiance measurements of cloud fields with even higher resolution data obtained from simultaneous Apollo photographs.
} 


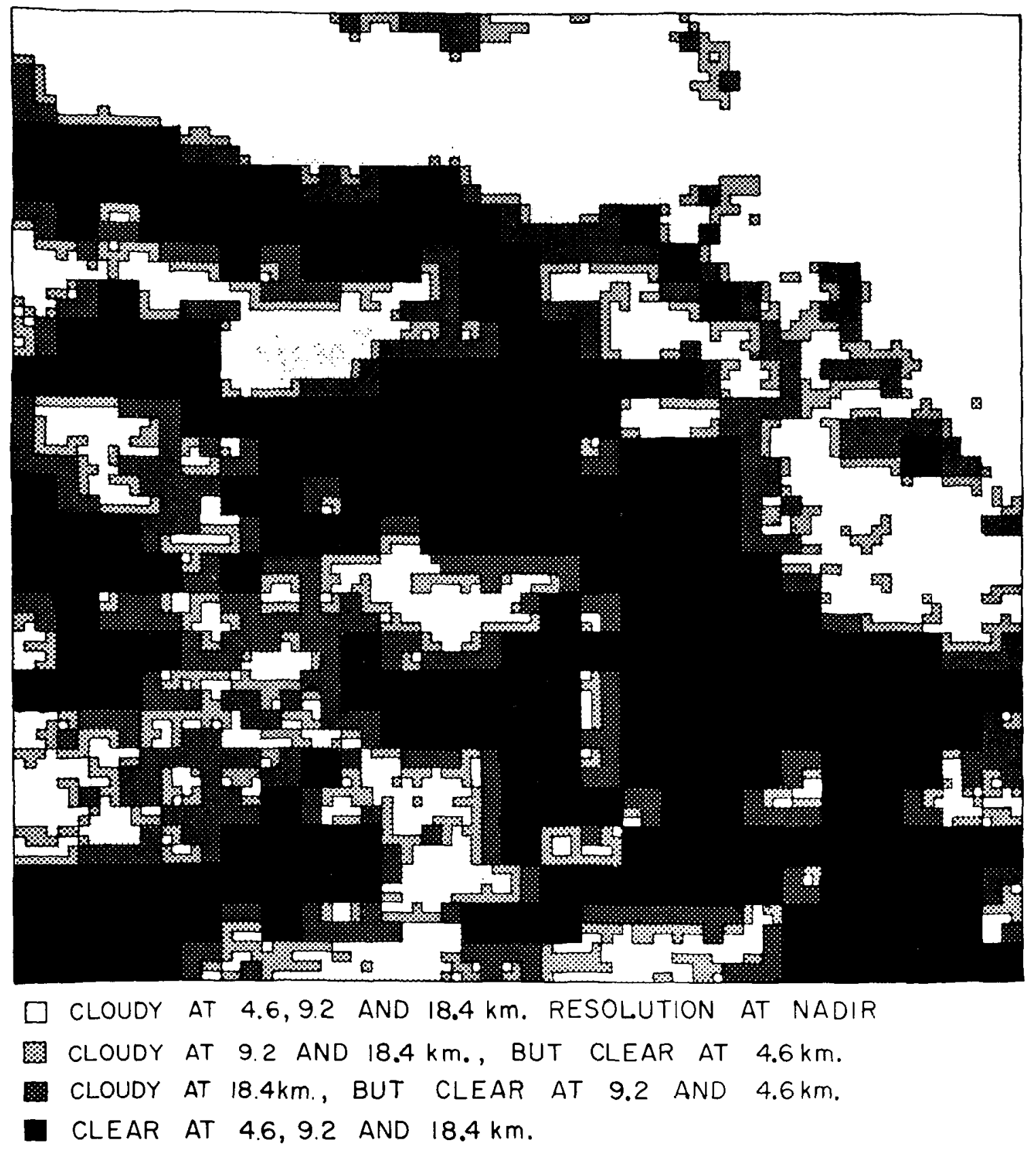

FIG. 3. Map of cloud cover for a high pressure zone cumulus and stratus in the subtropics for 20 January 1968, 1911:13 GMT, field 85 .

line plot of ATS-III. It should be noted that in the green channel (as stated before, this is the only one used in this experiment), the ocean is darker than the land. This implies that the difference in radiance levels between ocean and clouds is greater than that between land and clouds. For this reason and also because the radiance spread is less over the ocean than over the land (see Fig. 1), it is easier to make a threshold determination over the ocean.

The second method of determining the cloud-no cloud threshold is to plot the frequency at which a given radiance level occurs within a grid. If there is a reasonable amount of clear area within the grid, a large peak of roughly Gaussian shape will represent this clear area provided the clear area is homogeneous as is generally true over oceans. The point at which the curve starts to rise again, or a few radiance levels to the cloudy side of this clear area peak, is taken as the point where cloud interference is becoming important. This point is chosen as the threshold. Fig. 4 shows a radiance level-frequency plot for a cloudy area over the ocean. Land areas lack the homogeneity of oceans 


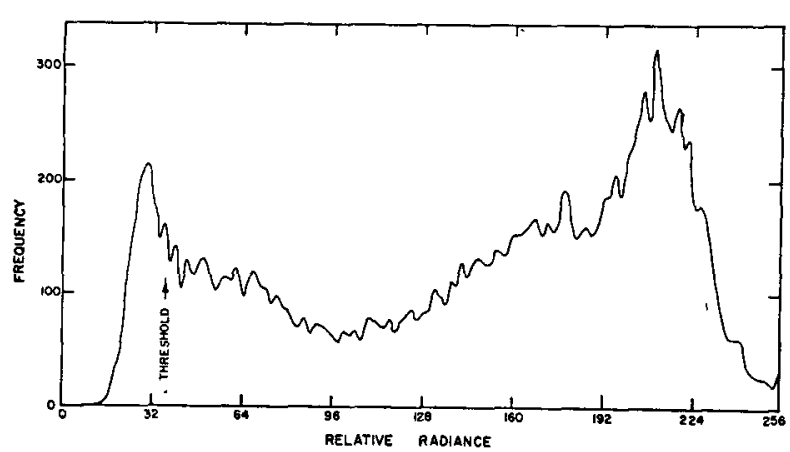

FIg. 4. Frequency plot of radiance levels over water, the raw data being corrected according to Hanson (1969), for 20 January 1968, 1911:31 GMT, field 94. Thirty-six was taken as threshold.

so this method of threshold determination is more difficult. Fig. 5 shows a radiance level-frequency plot for the average of eight grids over land.

In order to check the significance of an incorrect threshold, these eight grids were run with five different thresholds. The additional thresholds are indicated by small arrows in Fig. 5. Fig. 6 shows the average clear area of all eight grids in the matrix vs resolution for each of these thresholds. It is seen that the percentage clear area changes greatly with a change in threshold but that the shape of the curve remains about the same. Since it is difficult to obtain the threshold to within several radiance levels, an error might be expected in the values of percent clear area given in this paper; however, the change in percentage open area with resolution is more significant and also more accurate; it will, therefore, be emphasized in this paper.

In summary, cloud-no cloud thresholds can be determined from several methods. Two of the most basic have been used in this work. Particularly over land areas, the frequency distribution technique is difficult; thus, more reliance was placed on the line plot displays in selecting a threshold over land areas. In all cases, a conservative estimate of the thresholds was attempted; that is, when in doubt, the threshold was chosen to overestimate the cloudy regions.

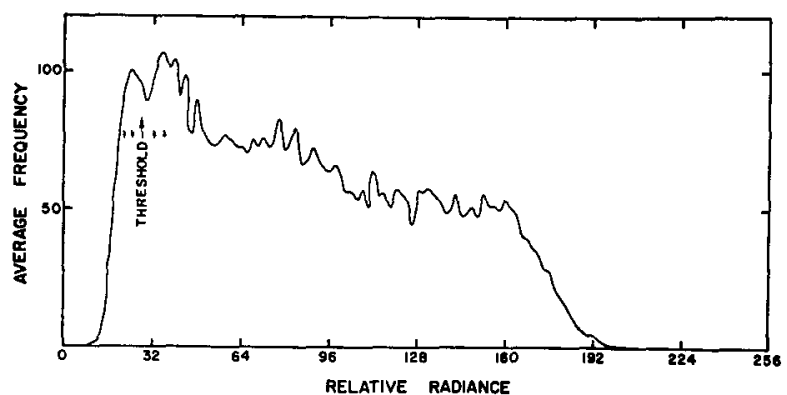

FIG. 5. Average frequency plot of radiance levels for eight grids over land, the raw data being corrected according to Hanson (1969), for 23 April 1968, 1714:10 GMT, matrix 1. Twentyeight was taken as threshold. Small arrows indicate additional thresholds used in Fig. 6.

\section{Results}

ATS measurements from 23 April 1968 (1714:10 GMT) and 20 January 1968 (1911:31 GMT) were chosen for this study. The grids for which the results are listed in this paper are in matrices 3 and 5 , and part of matrix 1 of Fig. 7, and fields $37-40,79-81,83-85$ and 87-95 of Fig. 8. Table 1 gives the percentage clear area using Method $\mathrm{B}$ for each of the grids mentioned above. These six matrices represent various meteorological conditions including extratropical fronts, cumulus and cumulonimbus over the Amazon, cumulus and stratus in the subtropical high pressure zone over the Pacific, and the intertropical covergence zone.

Figs. 9 and 10 compare the various grids using the clear areas derived from Method B normalized to the distribution density for each grid (defined as percent clear area at $4.6-\mathrm{km}$ resolution). Use of this parameter, distribution density, provides a base to compare grids having different meteorological conditions. Fig. 9 shows that although the warm sector has fewer clouds than the frontal band at $4.6-\mathrm{km}$ resolution, its percent clear area decreases faster with reduced resolution. This is because the clouds in the warm sector are a more irregular mixture of different types. Fig. 10, on the other hand, shows that when clouds of the same type are found in a number of grids, as is the case of high pressure zone cumulus and stratus, the decrease with resolution depends more on the distribution density. This suggests that for the same distribution density, a measure of variation in cloud type between two grids may be inferred from the rate of change of percent clear area with sensor resolution.

Figs. 11 and 12 summarize the results over various types of clouds and over regions with varying percent clear area at $4.6-\mathrm{km}$ resolution. Although the statistics are not large, the results, as anticipated, show that both parameters are important.

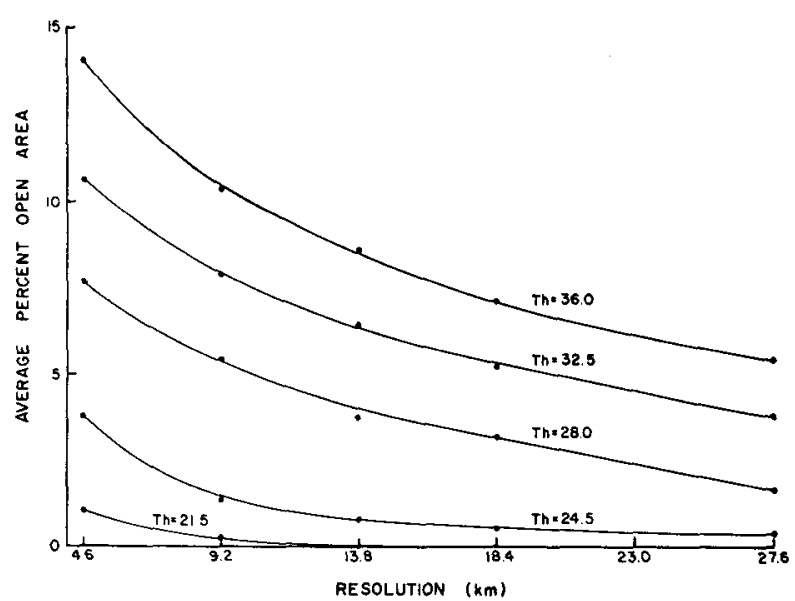

FIG. 6. Comparison of five thresholds for the same grids described in Fig. 5. The percent clear area is given at different spatial resolutions for the average of these grids. 


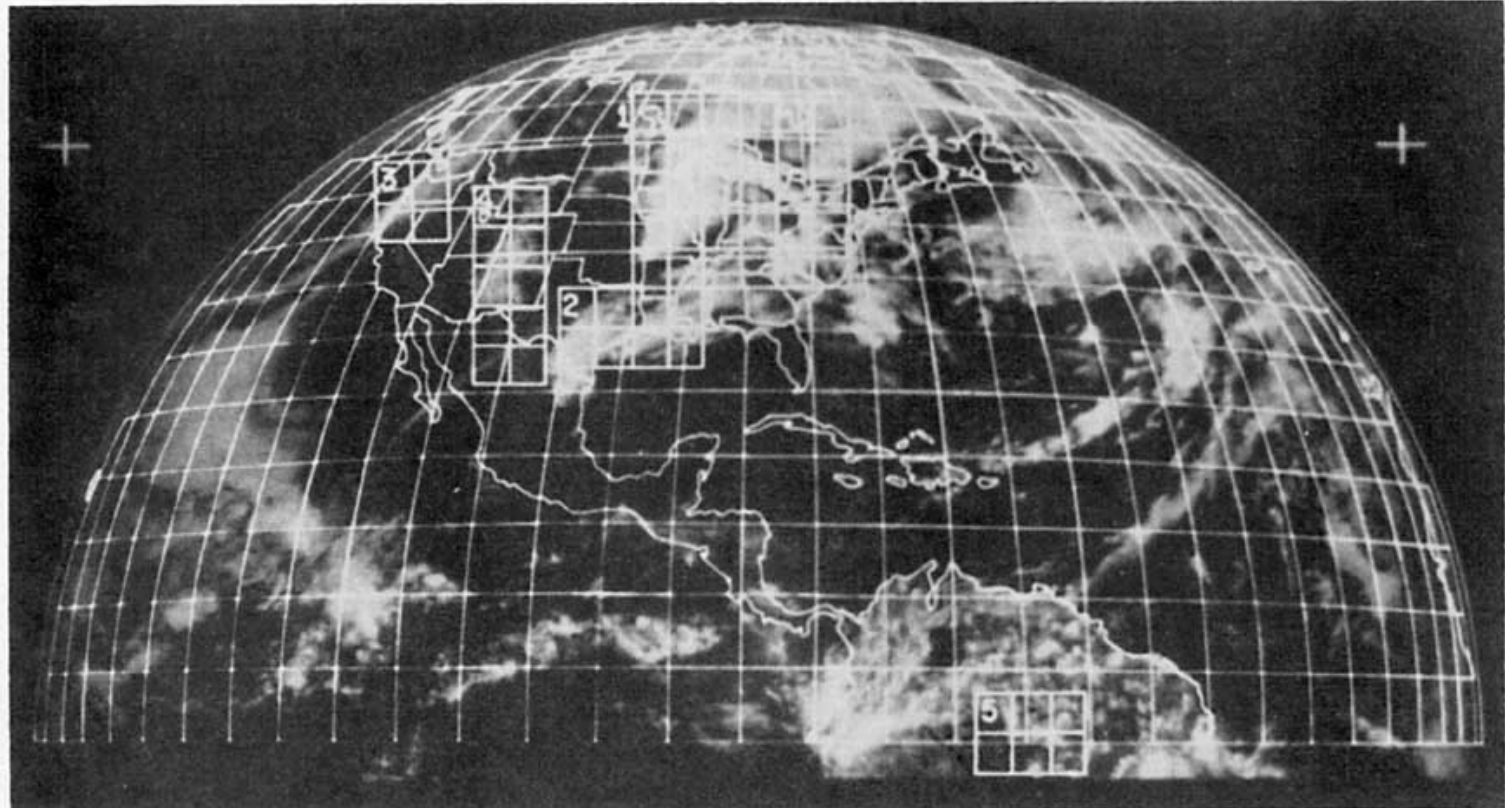

FIG. 7. ATS photograph for 23 April 1968, 1714:10. Investigated areas include matrix 1, rows 4 and 5, columns 2-5;

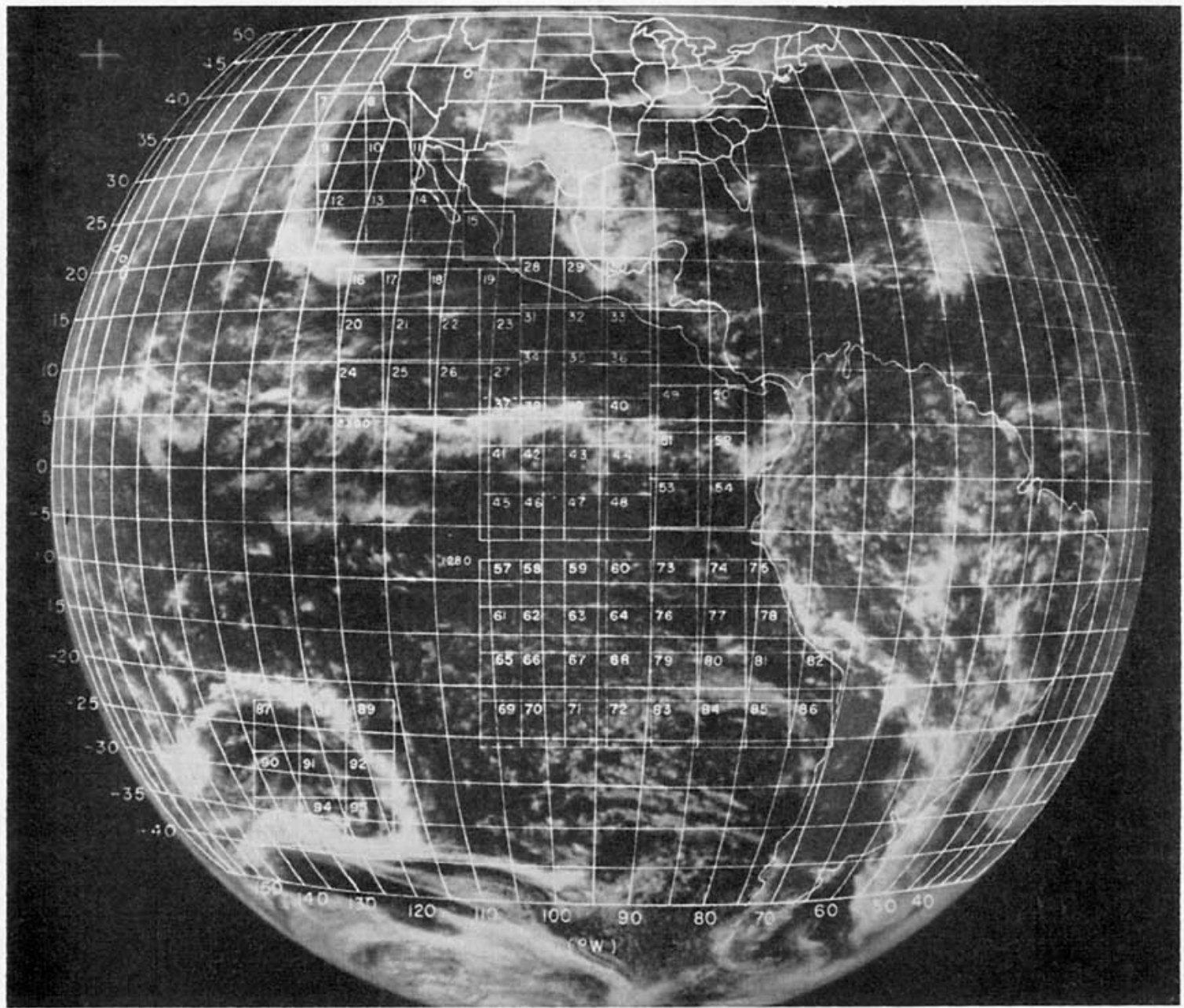

FIG. 8. ATS photograph for 20 January 1968, 1911:31. Investigated areas include fields $37-40,79-81,83-85$ and $87-95$. 
TABLE 1. Percentage clear area obtained by Method B. a. 23 April 1968 (1714:10 GMT)

\begin{tabular}{|c|c|c|c|c|c|c|}
\hline \multicolumn{7}{|c|}{ MATRIX 1} \\
\hline \multicolumn{7}{|c|}{$\begin{array}{c}\text { Extratropical front over midwestern United States } \\
\text { Resolution at nadir }(\mathrm{km})\end{array}$} \\
\hline & 4.6 & 9.2 & 13.8 & 18.4 & 23.0 & 27.6 \\
\hline Row & 11.36 & 9.00 & 7.25 & 5.7 & 4.17 & 4.00 \\
\hline Row $4 \mathrm{cl} 3$ & 16.17 & 10.00 & 5.8 & 4.00 & 2.78 & \\
\hline Row 4 cl 4 & 0 & 0 & 0 & 0 & & \\
\hline Row 4 cl 5 & 8.00 & 4.22 & 1.75 & 1.33 & 0 & 0 \\
\hline Row $5 \mathrm{cl} 2$ & 24.00 & 19.33 & 15.25 & 13.78 & 8.33 & 9.0 \\
\hline Row $5 \mathrm{cl} 3$ & 0.53 & 0.22 & 0 & 0 & 0 & 0 \\
\hline Row 5 d 4 & 1.14 & 0.11 & 0 & 0 & 0 & 0 \\
\hline Row 5 cl 5 & 0 & 0 & 0 & 0 & 0 & 0 \\
\hline \multicolumn{7}{|c|}{ MATRIX 3} \\
\hline \multicolumn{7}{|c|}{ Extratropical front over northwestern United States and ocean } \\
\hline Row $1 \mathrm{cl} 1$ & 80.3 & 74.22 & 67.75 & 60.89 & 56.94 & 53.00 \\
\hline Row $1 \mathrm{cl} 2$ & 8 & 6.33 & 4.7 & 3.11 & 2. & 1.0 \\
\hline Row $2 \mathrm{cl} 1$ & 18.89 & 12.67 & 9.50 & 6.67 & 5.56 & 3.0 \\
\hline Row 2 cl 2 & 40.53 & 23.11 & 10.25 & 4.89 & 1.39 & 0 \\
\hline \multicolumn{7}{|c|}{ MATRIX 5} \\
\hline \multicolumn{7}{|c|}{ Cumulus and cumulonimbus over Amazon } \\
\hline Row $1 \mathrm{cl} 1$ & 9.8 & 2.44 & 0.7 & 0 & 0 & 0 \\
\hline Row $1 \mathrm{cl} 2$ & 9.58 & 2.89 & 0.50 & 0 & 0 & 0 \\
\hline Row $1 \mathrm{cl} 3$ & 12.9 & 3.11 & 0.7 & 0 & 0 & 0 \\
\hline Row $2 \mathrm{cl} 1$ & 41.14 & 15.44 & 6.75 & 2.22 & 1.39 & 1.0 \\
\hline Row 2 cl 2 & 20.11 & 10.8 & 5.7 & 4.44 & 3.47 & 1. \\
\hline Row $2 \mathrm{cl} 3$ & 12.75 & 3.89 & 0.75 & 0.44 & 0 & 0 \\
\hline
\end{tabular}

b. 20 January 1968 (1911:31 GMT)

\begin{tabular}{|c|c|c|c|}
\hline \multicolumn{4}{|c|}{$\begin{array}{l}\text { Intertropical convergence zone } \\
\text { Resolution at nadir }(\mathrm{km})\end{array}$} \\
\hline & 4.6 & 9.2 & 18.4 \\
\hline $\begin{array}{l}\text { Field } 37 \\
\text { Field } 38 \\
\text { Field } 39 \\
\text { Field } 40\end{array}$ & $\begin{array}{r}7.85 \\
2.04 \\
4.06 \\
18.58\end{array}$ & $\begin{array}{r}5.44 \\
0.52 \\
1.40 \\
13.16\end{array}$ & $\begin{array}{l}3.04 \\
0 \\
0.32 \\
6.56\end{array}$ \\
\hline \multicolumn{4}{|c|}{ MATRIX 2} \\
\hline $\begin{array}{r}\text { Subtropical high } \\
\text { Field } 79 \\
\text { Field } 80 \\
\text { Field } 81 \\
\text { Field } 83 \\
\text { Field } 84 \\
\text { Field } 85\end{array}$ & $\begin{array}{c}\text { ressure } 2 \\
9.33 \\
6.75 \\
40.36 \\
36.70 \\
70.54 \\
65.00\end{array}$ & $\begin{array}{r}\text { ne cum } \\
2.96 \\
4.72 \\
34.32 \\
25.52 \\
60.64 \\
54.92\end{array}$ & $\begin{array}{c}\text { us and siratus } \\
0.32 \\
2.72 \\
27.36 \\
11.84 \\
44.64 \\
39.20\end{array}$ \\
\hline \multicolumn{4}{|c|}{ MATRIX 3} \\
\hline \multicolumn{4}{|c|}{ Extratropical front over southreest Pacific } \\
\hline $\begin{array}{l}\text { Field } 87 \\
\text { Field } 88 \\
\text { Field } 89 \\
\text { Field } 90 \\
\text { Field } 91 \\
\text { Field } 92 \\
\text { Field } 93 \\
\text { Field } 94 \\
\text { Field } 95\end{array}$ & $\begin{array}{r}61.15 \\
7.28 \\
27.47 \\
13.76 \\
24.20 \\
27.77 \\
0 \\
7.12 \\
14.60\end{array}$ & $\begin{array}{r}50.64 \\
3.60 \\
18.40 \\
4.64 \\
13.80 \\
19.56 \\
0 \\
4.60 \\
8.72\end{array}$ & $\begin{array}{l}38.08 \\
1.60 \\
9.76 \\
0.32 \\
6.24 \\
9.76 \\
0 \\
1.76 \\
3.52\end{array}$ \\
\hline
\end{tabular}

In order to see how the cloud cover in the areas studied changed in several hours, pictures were selected that were $2 \frac{3}{4}$ hr later on 23 April 1968 (1959:20) and $2 \frac{3}{4} \mathrm{hr}$ earlier on 20 January $1968(1629: 40)$. Since the selection of the threshold is very critical (see Fig. 5), it was decided to develop two selection methods that choose a threshold relative to the previous picture rather than use the threshold determination methods mentioned in the last section.

The first method uses the cosine of the sun angle at the subsatellite point as a factor for normalizing the thresholds to the pictures. In using this method, it is important that the camera and ground station gain settings are the same for the various pictures involved. Ideally, each sample of each picture should be normalized using a generalized bi-directional reflectance relationship, especially when angular sun and viewing condition vary a great deal between a pair of pictures. Such a technique is still under development.

The second method involves determining the fractional shift of the clear area peak of the radiance level frequency distribution (see Fig. 4). The peak is used because it is easier to find accurately than the point where cloud interference starts to dominate. [Hanson (1969) shows that over the ocean the clear area peak can be accurately predicted by forming a Gaussian curve from the data on the left-hand side of the peak, i.e., the side free from cloud interference.]

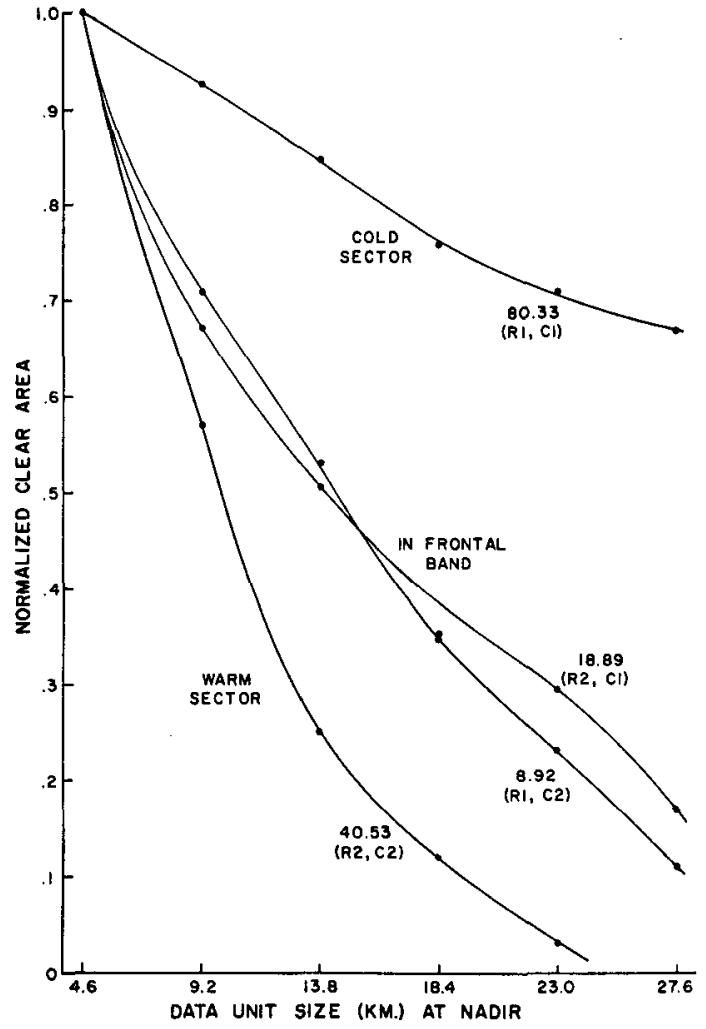

Frg. 9. Clear area vs resolution normalized to percent clear area at $4.6 \mathrm{~km}$ for an extratropical front over northwestern United States, 23 April 1968, 1714:10 GMT, matrix 3. Numbers indicate percent clear area at $4.6-\mathrm{km}$ resolution; and row and column locate grid within matrix. 
Results of the two methods agree with each other to within about three radiance levels (on a scale of $0-255$ ).

Fig. 13 shows the change with time of the percentage of clear observations for the various fields within the ITCZ and the high pressure zone cumulus and stratus region. The total of all three types of shading must be taken to get the percent clear area of $4.6-\mathrm{km}$ resolution, and the total of two shadings for the $9.2-\mathrm{km}$ resolution. Both of the regions studied for time variations are ones where air mass changes over a 3 -hr period are negligible. Despite this fact, a wide range in both relative and absolute changes of percent clear area over the same physical area are observed.

\section{An application to the radiometric temperature sounding problem}

The sounding problem involves a trade-off between two conflicting phenomena. The first involves the instrument. As the spatial resolution is increased, i.e., the angular field of view made smaller, the amount of energy received by a given telescope detector in a given spectral interval is decreased. Thus, to maintain the same statistical observational error, the instrument must look at the region of interest for a longer period of time. In' order to make the observational time as short as

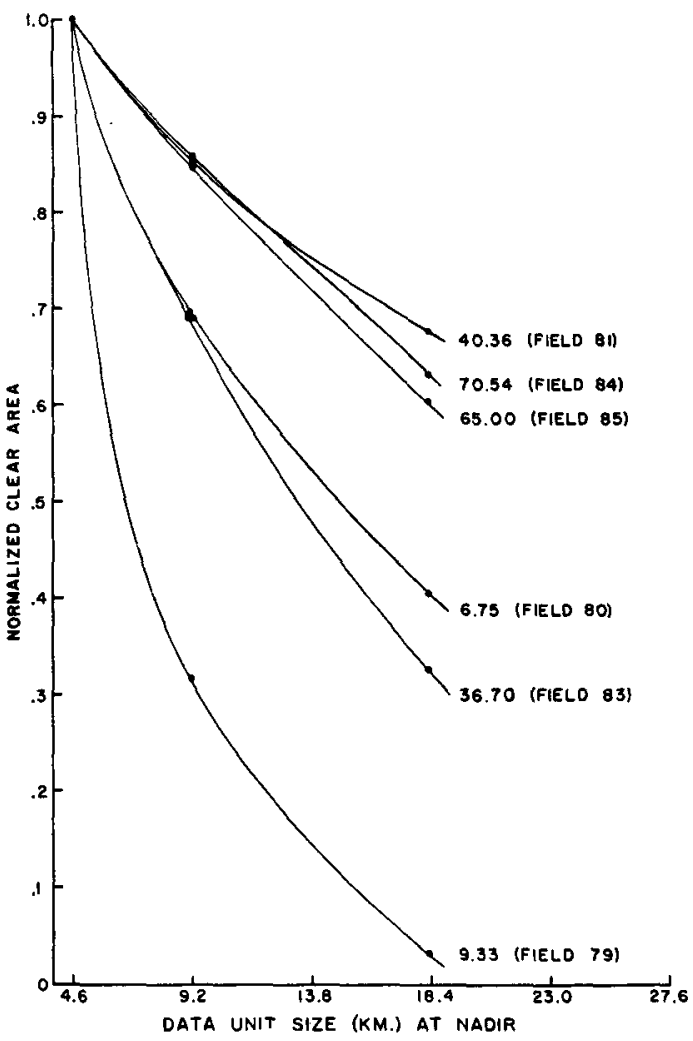

FIG. 10. Clear area vs resolution normalized to percent clear area at $4.6 \mathrm{~km}$ for subtropical high pressure zone cumulus and stratus on 20 January 1968, 1911:31 GMT, fields 79-81 and $83-85$. Numbers indicate percent clear area at $4.6-\mathrm{km}$ resolution.

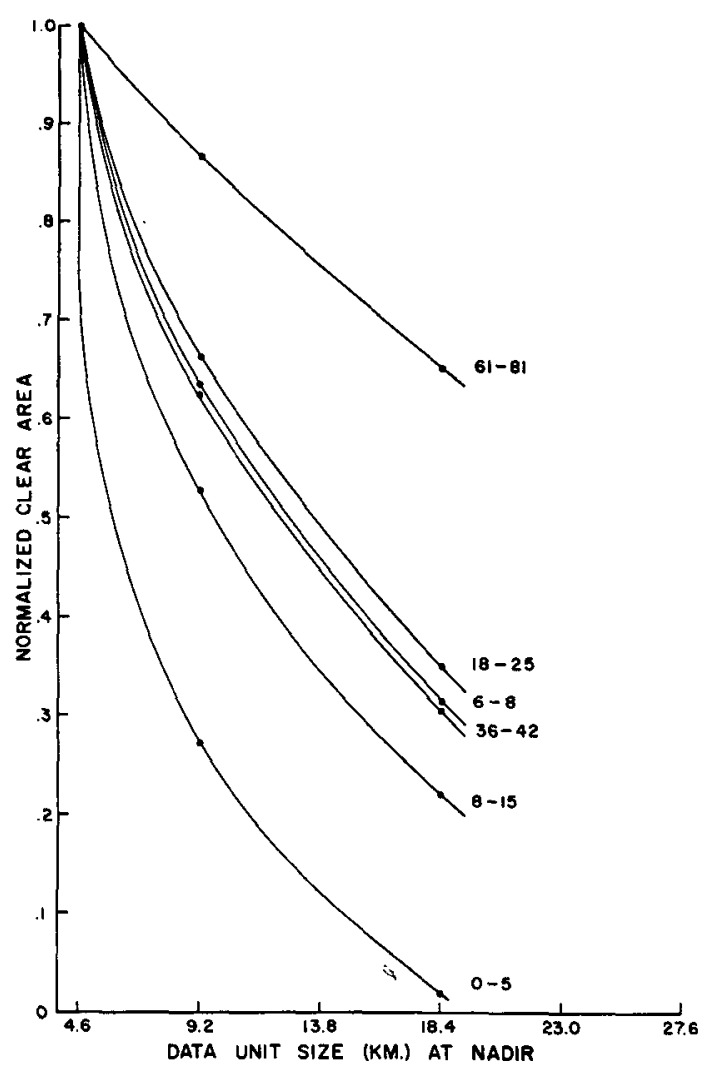

Fig. 11. Average clear area vs resolution normalized to percent clear area at $4.6 \mathrm{~km}$ for various degrees of cloudiness. Numbers indicate range of percent clear area at $4.6 \mathrm{~km}$.

possible, the instrument should have as low a spatial resolution as possible.

The second conflicting phenomenon involves the clouds themselves. As shown earlier, decreasing the spatial resolution (i.e., increasing the IFOV) decreases the percent of area which is seen as cloud-free. In many cases the cloud-free area goes to zero because there are no holes in the clouds larger than the field of view. Thus, the spatial resolution should be as high as possible. Since this latter phenomenon is more significant the greater the cloud cover, it must first be decided for what cloud cover a sounding instrument should be designed. Arking $e t$ al. ${ }^{4}$ are working with averages over cloudy and clear areas to determine the probability of finding clear areas in specified geographic regions as a function of the size of the clear area. The first areas studied have a rather large percentage clear area ( $69 \%$ on the average). The Wisconsin study deals only with cloudy areas since the soundings of greatest interest will be near meteorologically active situations (i.e., clouds); and if one can sound near clouds, certainly

1 Personal communication based on unpublished report, Cloud statistics affecting the sounding of the lower atmosphere from a satellite. Preliminary report, 25 July 1969, by Albert Arking, Frank Weinstein and Jay Fleischman, Institute for Space Studies, GSFC, NASA, New York, N. Y. 


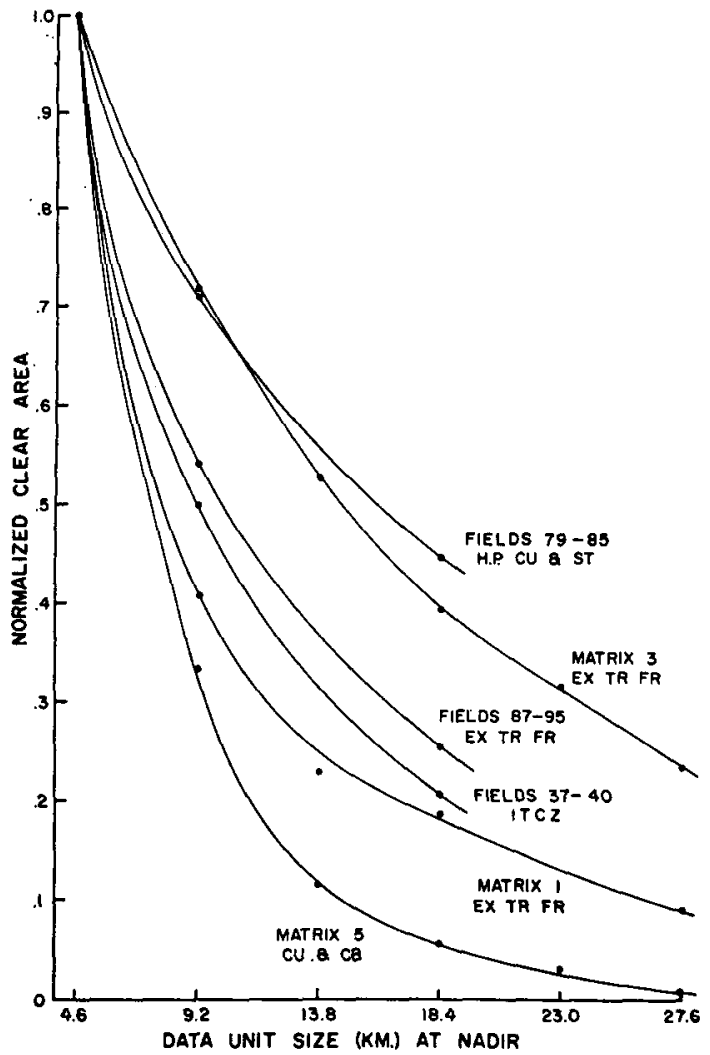

FIG. 12. Average clear area of each matrix vs resolution normalized to percent clear area at $4.6 \mathrm{~km}$.

one can sound in clear areas. In other words, a sounder should be designed for cloudy regions.

A sounding can be made by averaging the signals of all of the cloud-free instantaneous fields of view within a given area. (The GARP report recommends an area $400 \mathrm{~km}$ on a side.) Since numerical models require the data to represent the large-scale circulation, the input must be characteristic of the total area employed. The technique discussed in earlier sections fulfills this demand by considering many measurements over a relatively large area. Since the time base of the largescale variations is several days, it seems reasonable, if necessary, to spend several hours making a sounding. Thus, the earth can be scanned several times to gather the required number of radiance measurements. Temperature soundings inferred from such measurements will be asynoptic. However, they will also provide time derivatives of the temperature profile, data not obtained from a standard radiosonde network. The inherent time and space averaging must be done in conjunction with data interpolation and smoothing required by numerical models (GARP, 1969). Another channel with higher resolution having point-by-point correlation with the sounding channels (perhaps an infrared mapping channel) would be required to determine whether a given instantaneous field of view is cloud-free for the reasons stated earlier, i.e., the contrast in results between Methods $\mathrm{A}$ and $\mathrm{B}$.

Table 2, which gives the number of "soundable" grids in a given time at a given resolution, assumes a camera in geosynchronous orbit with 16-inch optics, spinning at $100 \mathrm{rpm}$ and stepping $0.2 \mathrm{mrad}$ each revolution. It is looking at a spectral band $20 \mathrm{~cm}^{-1}$ wide centered at $14 \mu \mathrm{m}$ with a detector having $D^{*} \approx 2 \times 10^{10} \mathrm{~cm} \mathrm{~Hz}^{\frac{1}{2}} \mathrm{~W}^{-1}$. The camera scans the whole earth enough times to give an error of $0.25 \mathrm{erg} \mathrm{cm}^{-2} \mathrm{sec}^{-1} \mathrm{ster}^{-1} \mathrm{~cm}$ over an area $400 \mathrm{~km}$ on a side at nadir (Krauss, 1969) in the areas of interest, i.e., those presented in Table 1. This type of instrument was studied because it can be retro-fitted to the camera system proposed for the Synchronous Meteorological Satellite (SMS). Table 2 shows that a temperature sounding radiometer is detector noise limited at smaller IFOV and "cloud noise" limited at larger IFOV. The times in this table can be decreased if only that part of the earth which contains dense clouds is scanned repeatedly. The conclusion to be drawn from this sparse data is that the ideal resolution for sounding in cloudy regions from a geosynchronous orbit is somewhere between 13.8 and $18.4 \mathrm{~km}$.

\section{Summary}

This study has examined the percentage of cloud-free area within defined regions. Furthermore, this parameter (percent clear area) was studied in relation to a varying IFOV of a possible satellite sensor. In addition, regions including various kinds of meteorological (i.e., cloud) conditions were studied at two times $2 \frac{3}{4} \mathrm{hr}$ apart.

It is difficult to generalize the results from this relatively small sample; more work of this kind is needed. However, one result seems evident: the percent clear area in a region decreases as the spatial resolution of the sensor is decreased. This result is expected. In addition, the rate of decrease of the measured parameter is increased as cloud "contaminants" smaller than the IFOV of the instrument are considered.

These preliminary results, based on actual measurements, from one of the highest resolution satellite sensors available to date (the ATS multicolor spin-scan cloud camera), may be interpreted and used for various applications. In the present study, meteorologically active regions have purposely been chosen as areas of interest. This choice was made since we are interested in assessing the ability of an infrared sounder on a

TABLE 2. Number of grids which can be sounded in a given time at a given resolution. The first 18 grids of Table 1 are used.

\begin{tabular}{rrrrr}
\hline $\begin{array}{l}\text { Resolution } \\
(\mathrm{km})\end{array}$ & $<1 \mathrm{hr}$ & $<2 \mathrm{hr}$ & $<4 \mathrm{hr}$ & $<6 \mathrm{hr}$ \\
\hline 9.2 & 2 & 5 & 9 & 11 \\
13.8 & 9 & 10 & 13 & 14 \\
18.4 & 10 & 11 & 11 & 11 \\
23.0 & 9 & 9 & 9 & 9 \\
27.6 & 7 & 7 & 7 & 7 \\
\hline
\end{tabular}




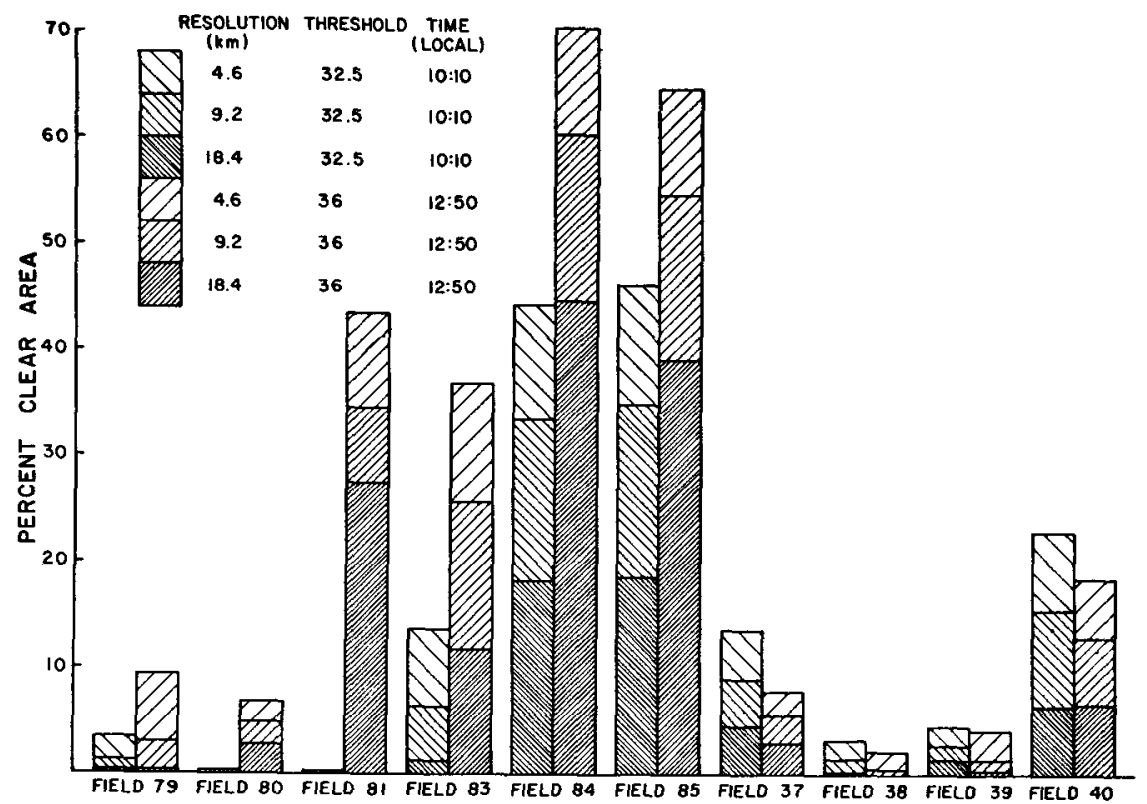

FIG. 13. Change in percent clear area in a few hours for a subtropical high pressure zone cumulus and stratus (fields 79-85) and the intertropical convergence zone (fields 37-40) on 20 January 1968, 1629:40 and 1911:31.

geosynchronous satellite to obtain vertical temperature profiles in and near these areas of prime meteorological interest. These sparse data, when combined with other studies of instrument design and accuracy to optimize an infrared sounding system, indicate that a good spatial resolution at nadir for a sounder is about $15 \mathrm{~km}$. This is equivalent to about $0.4 \mathrm{mrad}$ angular resolution from geosynchronous orbit.

Of general interest is the indication that clouds smaller than the instrument IFOV are very important. This indicates that determining a radiance level threshold in a visible channel is not sufficient by itself to decide whether small clouds are within the instrument's IFOV. Studies similar to the present paper should use simultaneous near-infrared and thermal infrared data to see if some combination of threshold criteria in several spectral channels can detect cloud contamination with better precision. Furthermore, the sensor used to detect cloud effects should have higher spatial resolution than any sounding instrument.

Acknowledgments. We thank Mr. Eric Smith for developing the computer programs; Mr. Robert Krauss, Dr. Kirby Hanson and Prof. Verner Suomi for their helpful discussions; and Miss Betty Erwin for typing the manuscript.

\section{REFERENCES}

COSPAR, 1969: Systems possibilities for an early GARP experiment. Working Group VII Rept. to Joint Organizing Committee, p. 50.

GARP, 1969: Plan for U. S. Participation in the Global Atmospheric Research Program. Washington, D. C., National Academy of Sciences, 16-17.

Hanson, Kirby J., 1969: Applications for bispectral radiance measurements from a satellite. Ph.D. thesis, Dept. of Meteorology, University of Wisconsin.

Krauss, Robert, 1969: Performance analysis of a proposed high accuracy geostationary sounding and imaging system. Annual Report on Contract NAS 511542 (in press). Available from Space Science and Engineering Center, 1225 West Dayton Street, Madison, Wis.

Salomonson, Vincent V., 1969 : Cloud statistics in earth resources technology satellite (ERTS) mission planning. GSFC preprint X-622-69-386, available from Goddard Space Flight Center, Greenbelt, $\mathrm{Md}$.

Smith, William L., 1968: An improved method for calculating tropospheric temperature and moisture from satellite radiometer measurements. Mon. Wea. Rev., 96, 387-396.

Thomsen, R., R. Parent and V. Suomi, 1969: ATS-I spin scan camera. Weather Motions From Space, Madison, University of Wisconsin Press (in press).

Vonder Haar, T. H., 1969 : Meteorological applications of reflected radiance measurements from ATS-I and ATS-III. $J$. Geophys. Res., 74, 5404-5412. 